\title{
Comparative transcriptomic analysis of thermally stressed Arabidopsis thaliana meiotic recombination mutants
}

\author{
Jiyue Huang ${ }^{1+}\left(\mathbb{D}\right.$, Hongkuan Wang ${ }^{2 \dagger} \mathbb{B}$, Yingxiang Wang ${ }^{2^{*}}$ (D) and Gregory P. Copenhaver ${ }^{1,3^{*}}$ (D)
}

\begin{abstract}
Background: Meiosis is a specialized cell division that underpins sexual reproduction in most eukaryotes. During meiosis, interhomolog meiotic recombination facilitates accurate chromosome segregation and generates genetic diversity by shuffling parental alleles in the gametes. The frequency of meiotic recombination in Arabidopsis has a U-shaped curve in response to environmental temperature, and is dependent on the Type I, crossover (CO) interference-sensitive pathway. The mechanisms that modulate recombination frequency in response to temperature are not yet known.
\end{abstract}

Results: In this study, we compare the transcriptomes of thermally-stressed meiotic-stage anthers from msh4 and mus81 mutants that mediate the Type I and Type II meiotic recombination pathways, respectively. We show that heat stress reduces the number of expressed genes regardless of genotype. In addition, msh4 mutants have a distinct gene expression pattern compared to mus81 and wild type controls. Interestingly, ASY1, which encodes a HORMA domain protein that is a component of meiotic chromosome axes, is up-regulated in wild type and mus 81 but not in msh4. In addition, SDS the meiosis-specific cyclin-like gene, DMC1 the meiosis-specific recombinase, SYN1/REC8 the meiosis-specific cohesion complex component, and SWI1 which functions in meiotic sister chromatid cohesion are up-regulated in all three genotypes. We also characterize 51 novel, previously unannotated transcripts, and show that their promoter regions are associated with A-rich meiotic recombination hotspot motifs.

Conclusions: Our transcriptomic analysis of msh4 and mus81 mutants enhances our understanding of how the Type I and Type II meiotic CO pathway respond to environmental temperature stress and might provide a strategy to manipulate recombination levels in plants.

Keywords: Heat stress, Meiotic recombination, MUS81, MSH4, ASY1, RNA-Seq

\footnotetext{
* Correspondence: yx_wang@fudan.edu.cn; gcopenhaver@bio.unc.edu ${ }^{\dagger}$ Jiyue Huang and Hongkuan Wang contributed equally to this work.

${ }^{2}$ State Key Laboratory of Genetic Engineering and Collaborative Innovation Center of Genetics and Development, Ministry of Education Key Laboratory of Biodiversity Sciences and Ecological Engineering, Institute of Plant Biology, School of Life Sciences, Fudan University, Shanghai, China

'Department of Biology and the Integrative Program for Biological and Genome Sciences, University of North Carolina at Chapel Hill, Chapel Hill, North Carolina, USA

Full list of author information is available at the end of the article
}

(C) The Author(s). 2021 Open Access This article is licensed under a Creative Commons Attribution 4.0 International License, which permits use, sharing, adaptation, distribution and reproduction in any medium or format, as long as you give appropriate credit to the original author(s) and the source, provide a link to the Creative Commons licence, and indicate if changes were made. The images or other third party material in this article are included in the article's Creative Commons licence, unless indicated otherwise in a credit line to the material. If material is not included in the article's Creative Commons licence and your intended use is not permitted by statutory regulation or exceeds the permitted use, you will need to obtain permission directly from the copyright holder. To view a copy of this licence, visit http://creativecommons.org/licenses/by/4.0/ The Creative Commons Public Domain Dedication waiver (http://creativecommons.org/publicdomain/zero/1.0/) applies to the data made available in this article, unless otherwise stated in a credit line to the data. 


\section{Background}

Meiosis consists of a pair of cell divisions that produce gametes in sexually reproducing species. Prophase I of meiosis is distinct compared to mitosis because homologous chromosomes pair and reciprocally exchange DNA in a process called recombination. In most species, each pair of homologs must experience at least one exchange, or crossover $(\mathrm{CO})$, in order to segregate properly at end of the first meiotic division. In the absence of a $\mathrm{CO}$, chromosomes segregate randomly, resulting in chromosome number imbalances, or aneuploidy, in the gametes which, in turn, can result in developmental defects or lethality in progeny. Regulation of the frequency and distribution of COs in the genome has been studied extensively [1] and more recently, advances have been made in our understanding of how environmental conditions and stressors influence those regulatory mechanisms [2-4].

Numerous studies over the last century have demonstrated that environmental stressors, including thermal, nutrient, and drought stress can modulate $\mathrm{CO}$ frequencies in animals, fungi and plants $[1,5,6]$. The effect of temperature on meiotic recombination has been examined in several systems with results that suggest speciesspecific and complex regulatory mechanisms. In the model plant Arabidopsis thaliana for example, meiotic recombination exhibits a U-shaped response curve corresponding to variation from low to high environmental temperatures [3]. In other species, like Hyacinthus orientalis and Oryza sativa, meiotic recombination also increases with temperature, but in Endymion nonscriptus and Rhoeo spathacea it decreases [1, 5, 6]. In Hordeum vulgare (barley), a shift from $15^{\circ} \mathrm{C}$ to $30^{\circ} \mathrm{C}$ resulted in an incremental increase in male (but not female) $\mathrm{CO}$ frequencies, but also a distinct shift from proximal to distal COs along the chromosome arms [7]. At even higher temperatures, meiotic mechanisms begin to fail resulting in infertility $[8,9]$.

In plants, COs are divided into Type I, which are subject to a regulatory phenomenon known as crossover interference that inhibits closely spaced COs, and Type II which are insensitive to interference. MSH4 is a member of the ZMM (ZIP4, MSH4/5, MER3) group of proteins [10] that mediate the interference sensitive Type I pathway. MSH4 encodes a meiosis-specific member of the MutS family of proteins responsible for DNA mismatch repair (MMR) in eukaryotes and prokaryotes. However, unlike other MutS family members, MSH4 does not show MMR activity. Instead, it is required for reciprocal meiotic recombination and proper homologous chromosome segregation [11]. MUS81, an endonuclease that resolves recombination intermediates, is required for Type II interference insensitive COs in most organisms [12]. In Arabidopsis, MSH4 mediates about $85 \%$ of COs, while MUS81 is responsible for $15 \%[11,12]$. Type I COs can also be quantified, and compared to genetic $\mathrm{CO}$ frequencies, using immunolocalization with antibodies to the MutL homolog, MLH3 [13]. Using this technique Phillips et al., showed that the high temperature-induced increase in the barley male genetic map was not accompanied by an increase in Type I COs [7], suggesting the hyper-recombinant response is instead mediated by the Type II pathway. These results stand in contrast to two recent studies in Arabidopsis thaliana that show a similar thermal-stress induced hyperrecombination phenotype, but use mutant analysis to demonstrate that the response is mediated by the Type I rather than Type II pathway [2, 3]. Thus, while the ability to modulate $\mathrm{CO}$ frequency in response to thermal stress is conserved between monocots and dicots, the specific pathways employed may differ significantly. Moreover, we know little about the gene products that sense environmental cues, and transduce them to the meiotic recombination machinery, although alteration of synaptonemal complex (SC) structure, modulation of chromatin states, and changes in the epigenetic landscape have been suggested as intriguing candidates $[1,5,6]$.

To identify genes involved in mediating the thermal stress induced hyper-recombination phenotype, we performed a comparative analysis of the transcriptomes of meiotic stage anthers from wild type (WT; Col-0), msh4 and mus81 plants grown under control and thermallystressed conditions. We found that heat stress reduces the number of gene expressed in all genotypes. WT and mus81 plants have similar thermal-stress induced expression profiles which are distinct compared to $m s h 4$. Interestingly, the gene encoding the HORMA domaincontaining chromosome axis protein $A S Y 1$ is upregulated in wild type and mus 81 but not in msh4. Additionally, we found 51 novel, unannotated transcripts that are associated with previously defined A-rich meiotic recombination hotspot motifs. Our transcriptomic analysis of meiotic Type I and Type II CO pathway mutants in temperature-stress conditions sheds new light on how abiotic factors regulate meiotic recombination in plants.

\section{Results \\ mRNA-Seq characteristics of meiotic recombination pathway mutants}

Previous reports from our lab and others revealed that the increased meiotic crossover frequencies observed in Arabidopsis grown at elevated temperatures are mediated by the Type I interference sensitive pathway [2, 3]. To identify genes potentially involved in the hyperrecombinant phenotype, we collected stage 4-7 anthers that contain male meiocytes from leptotene to tetrad stage [14] from WT (Col-0), mus81, and msh4 plants. We collected two biological replicates for each genotype grown at $20^{\circ} \mathrm{C}$ and $28^{\circ} \mathrm{C}$, then sequenced the 12 mRNA 
libraries. A total of $690,371,408$ raw reads were retrieved from the 12 mRNA-Seq datasets with an average of 53 million mapped reads per dataset and a 93\% average mapping rate (Supplementary Table 1). The biological replicates for each genotype-temperature combination had a minimum 0.92 correlation coefficient (Supplementary Table 2) indicating high reproducibility among our datasets. We examined the number of expressed genes in each condition with a threshold equal to or greater than 1 TPM (Transcripts Per Kilobase Million). At $20^{\circ} \mathrm{C}$, WT, mus 81 and $m s h 4$ have 20,489, 20,755 and 20 , 116 expressed genes, respectively (Fig. 1a). After $28^{\circ} \mathrm{C}$ heat stress treatment, the number of expressed genes decreased in all three genotypes (Fig. 1a). Both WT and a

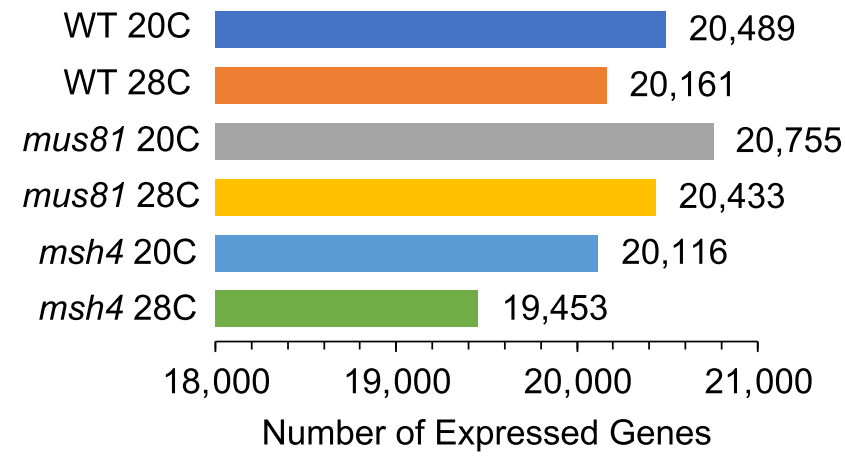

b

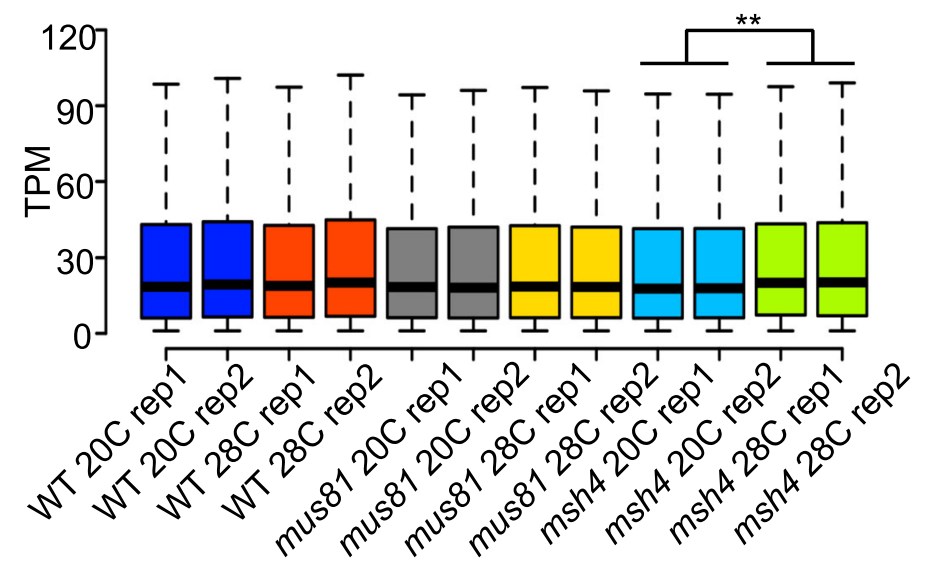

C

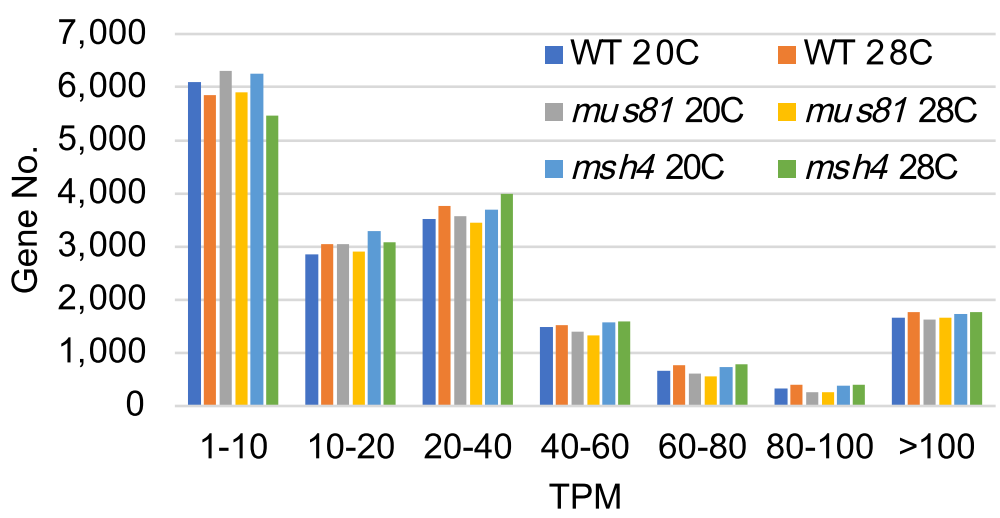

Fig. 1 Characteristics of the heat-stressed and control transcriptomes in WT, mus81 and msh4. a. The number of expressed genes in the six experimental samples. b. Gene expression value in all samples with two biological replicates (**P value $<2.2 \mathrm{e}-16$ from Mann-Whitney test). $\mathbf{c}$. Expressed genes grouped from low to high from all the six experimental samples 
mus 81 have $1.6 \%$ fewer expressed genes, while msh4 has $3.3 \%$ fewer. We also compared the average level of gene expression in two biological replicates for each genotype at $20^{\circ} \mathrm{C}$ and $28^{\circ} \mathrm{C}$. The TPM values do not differ significantly for WT (18.4 and 19.4 at $20^{\circ} \mathrm{C}$ verus 18.8 and 20.1 at $28^{\circ} \mathrm{C}$, Mann-Whitney test, $P$ value $\left.=0.05\right)$ and mus81 (18.4 and 18.1 at $20^{\circ} \mathrm{C}$ verus 18.5 and 18.4 at $28^{\circ} \mathrm{C}$, Mann-Whitney test, $P$ value $=0.18$ ), but did for msh4 $\left(17.7\right.$ and 17.9 at $20{ }^{\circ} \mathrm{C}$ versus 20.0 and 20.2 at $28^{\circ} \mathrm{C}$; Mann-Whitney test, $P$ value $<2.2 \mathrm{e}-16$ ) (Fig. $1 \mathrm{~b}$ ), suggesting that heat stress decreases the number of expressed genes in msh4 but increases the average strength of gene expression. We also compared the number of expressed genes in seven TPM value groups from low to high and found that all treatments and genotypes are similarly distributed, and that the group with TPM values between 1 and 10 is the largest (Fig. 1c). We then examined the overlap of expressed gene in WT, mus 81 , and $m s h 4$ at $20^{\circ} \mathrm{C}$ and $28^{\circ} \mathrm{C}$. At least $90 \%$ $(18,398)$ of expressed genes are shared between all datasets, and 63 genes are shared by only WT and mus 81 at $28{ }^{\circ} \mathrm{C}$ (Supplementary Figure 1a). Only one enriched GO term, phospholipase $\mathrm{C}$ activity from Molecular Function (GO: $0004629, \quad F D R=0.02$, by AT3G03530 and
AT3G03540) was detected among these 63 genes, no meiotic recombination related genes were found, and most of the 63 genes have relatively low gene expression (Supplementary Figure 1b).

\section{WT and mus81 have similar differential gene expression profiles}

By comparing expression levels of genes at $28^{\circ} \mathrm{C}$ to those at $20^{\circ} \mathrm{C}$ in each genotype, we found 2922, 2363 and 4009 differentially expressed genes (DEGs) from WT, mus81 and msh4, respectively. Those include 1196 and 1727 up- and down-regulated genes in WT, 1015 and 1348 in mus81, and 835 and 3174 in $m s h 4$. We analyzed the intersections of the up- and down-regulated DEGs (Fig. 2a, b) to determine whether any of the genotypes had similar profiles. On a proportional basis, WT and mus 81 share $22 \%$ of their up-regulated DEGs and $25 \%$ of their down-regulated DEGs, WT and msh4 share 19 and 19\% respectively, and mus 81 and $m s h 4$ share 13 and $12 \%$ respectively. WT and mus 81 share significantly more up-regulated DEGs compared to mus 81 and msh4 $\left(\chi^{2}=36.78, P\right.$ value $\left.=1.32 \mathrm{E}-09\right)$, and not significantly more to WT and msh $4\left(\chi^{2}=3.56, P\right.$ value $\left.=0.06\right)$. Similarly, WT and mus 81 share significantly more down-

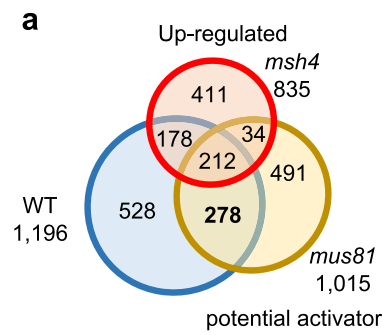

b

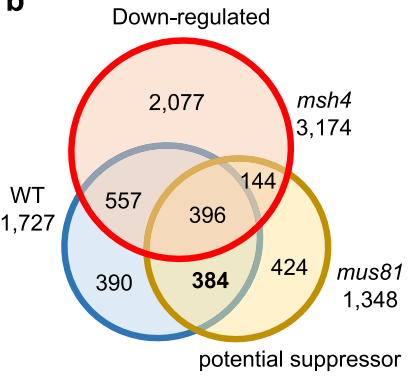

C

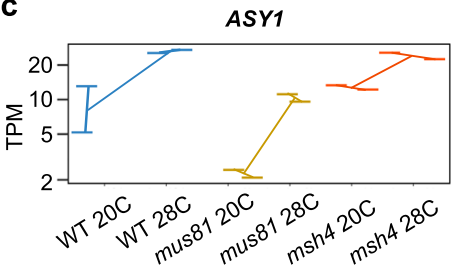

d
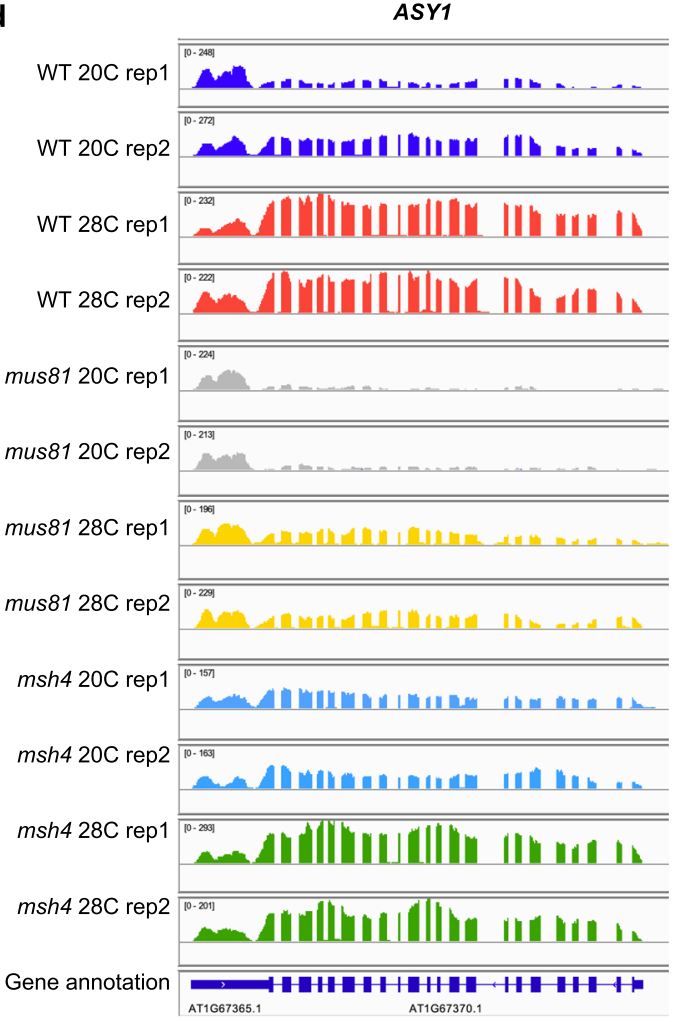

Fig. 2 Characteristics of the differentially expressed genes (DEGs) from WT, mus 81 and msh 4 Arabidopsis grown under $20^{\circ} \mathrm{C}$ and $28^{\circ} \mathrm{C}$ conditions. a. Venn diagram showing the common and specific up-regulated DEGs under heat stress among WT, mus81 and msh4. b. Venn diagram showing the common and specific down-regulated DEGs under heat stress among WT, mus81 and msh4. c. Expression of ASY1 from WT, mus81 and msh4 grown under $20^{\circ} \mathrm{C}$ and $28^{\circ} \mathrm{C}$ conditions. d. Snapshot showing that ASY1 is an up-regulated DEG in WT and mus81 but not in msh4 
regulated DEGs compared to WT and $m s h 4\left(\chi^{2}=24.51\right.$, $P$ value $=7.38 \mathrm{E}-07)$, as well as mus81 and msh4 $\left(\chi^{2}=\right.$ $158.02, P$ value $<2.2 \mathrm{E}-16$ ). It should be noted that disruption of the Type I CO pathway in msh 4 compromises but does not abolish pollen development and fertility, while WT and mus 81 are fertile, and that these differences in phenotype likely influence the differential gene expression patterns. Taken together, these data suggest that WT and mus81 have the most similar DEG profiles of the genotypes examined (Fig. 2a).

We performed GO enrichment analysis to explore the characteristics of the DEGs from WT, mus 81 and msh4 grown at $20^{\circ} \mathrm{C}$ and $28{ }^{\circ} \mathrm{C}$. Among the up-regulated DEGs, 45 Biological Process (BP), 7 Molecular Function (MF) and 3 Cellular Component (CC) GO terms were enriched in WT; 71, 19 and 7 in mus81; and 36, 0 and 0 in msh4 (Supplementary Data 1). Several GO terms enriched among the 1196 DEGs from WT and 1015 from mus 81 are not enriched in the 835 DEGs from msh4 including: 22 BP GO terms including cellular response to decreased oxygen levels (GO:0036294), response to heat (GO:0009408), protein folding (GO: 0006457), homologous chromosome segregation (GO: 0045143), and chromosome organization involved in meiotic cell cycle (GO:0070192), 4 MF GO terms including unfolded protein binding (GO:0051082) and 2 CC GO terms including anchored component of membrane (GO:0031225) (Fig. 3 and Supplementary Data 1). Among the down-regulated DEGs, there were $26 \mathrm{BP}, 4$ MF and 0 CC GO terms enriched in WT; 34, 18 and 8 in mus81; and 31, 13 and 9 in msh4 (Supplementary Data 1$)$. Only 6 BP GO terms, including response to absence of light (GO:0009646) and glucose import (GO: 0046323), and $1 \mathrm{MF}$ (carboxylic ester hydrolase activity; GO:0052689) are enriched in the down-regulated DEGs from WT and mus81 (Supplementary Data 1 and Supplementary Figure 2). We noticed that $m s h 4$ has the most down-regulated DEGs, and enriched GO terms not shared with WT or mus 81 that relate to male gametogenesis, such as pollen wall assembly (GO:0010208), pollen sperm cell differentiation (GO:0048235) and pollen exine formation (GO:0010584) (Supplementary Figure 2A). As mentioned above, this may be a consequence of the compromised pollen development phenotype observed in msh4.

To determine whether the up- and down-regulated DEGs from WT, mus81 and msh4 display any pattern along the 5 Arabidopsis chromosomes we plotted their density as a function of physical position (Fig. 4a, c). We did not detect any global patterns in distribution: upregulated DEGs from WT and mus 81 have a correlation coefficient of 0.46 , while WT and $m s h 4$ have a correlation coefficient of 0.42 . Similarly, the down-regulated DEGs have a correlation coefficient of 0.51 between WT and mus 81 and 0.43 between WT and msh4. To examine the local distribution patterns of DEGs, we divided each chromosome into 12 euchromatic segments of equal length and one heterochromatic segment and tested whether the number of WT DEGs in each segment differs significantly from those of mus 81 or $m s h 4$. The number of up-regulated DEGs from mus 81 and msh4 do not differ significantly from WT (Fig. 4b). Interestingly, two segments on the north arms of chromosome 1 and 4 show significantly more downregulated DEGs in msh4 than in WT (Fig. 4d). Overall, the distribution of DEGs does not differ dramatically among the genotypes but is consistent with WT and mus 81 being more similar to one another than msh4.

\section{GO terms enriched in the DEGs shared by WT and mus 81}

Exposure to elevated temperature results in an increase in Type I (MSH4-dependent) COs in Arabidopsis [2, 3]. To determine if that phenotype is correlated with changes in gene expression, we looked for DEGs shared between WT and mus81 (in which the Type II pathway is inactive) but not msh4. We found 278 and 384 downregulated DEGs that may be implicated in modulating the activity of the two pathways (Fig. 2a, b). GO analysis of the 278 up-regulated DEGs reveals enrichment of 3 BP GO terms: response to heat (GO:0009408), protein folding (GO:0006457) and response to toxic substance (GO:0009636), and 1 MF GO term: unfolded protein binding (GO:0051082) (Fig. 3d and Supplementary Data 2). Among the 384 down-regulated DEGs, 14 BP GO terms were enriched including: cellular response to decreased oxygen levels (GO:0036294), response to absence of light (GO:0009646), response to water deprivation (GO:0009414), and circadian rhythm (GO: 0007623) (Fig. 3d and Supplementary Data 2).

\section{ASY1 is exclusively up-regulated in WT and mus 81 but not in $m s h 4$}

We were interested in whether meiosis related genes are differentially expressed under heat stress. To this end, we examined the expression patterns of 148 genes that have been previously reported to have meiotic function (Supplementary Data 3). A heatmap comparison of these genes does not reveal any significant global difference in expression levels at $28^{\circ} \mathrm{C}$ compared to $20^{\circ} \mathrm{C}$ in WT, mus 81 or msh4 (Fig. 5). To our surprise, $A S Y 1$ and $A S Y 2$ are the only consistently up-regulated DEGs exclusively shared by WT and mus 81 but not msh4 (Fig. 2c, d; Supplementary Figure $3 \mathrm{a}, \mathrm{c})$ (ASY1 and ASY2 show an increase below the 2-fold cut-off at $28^{\circ} \mathrm{C}$ compared to $20^{\circ} \mathrm{C}$ in $m s h 4$, in contrast to 2.9 and 2.3-fold increases in WT, and 4.6 and 3.5-fold increases in mus81). ASY1 encodes a component of the chromosome axis that forms along the length of replicated sister chromatids during meiosis [15], and is required for 


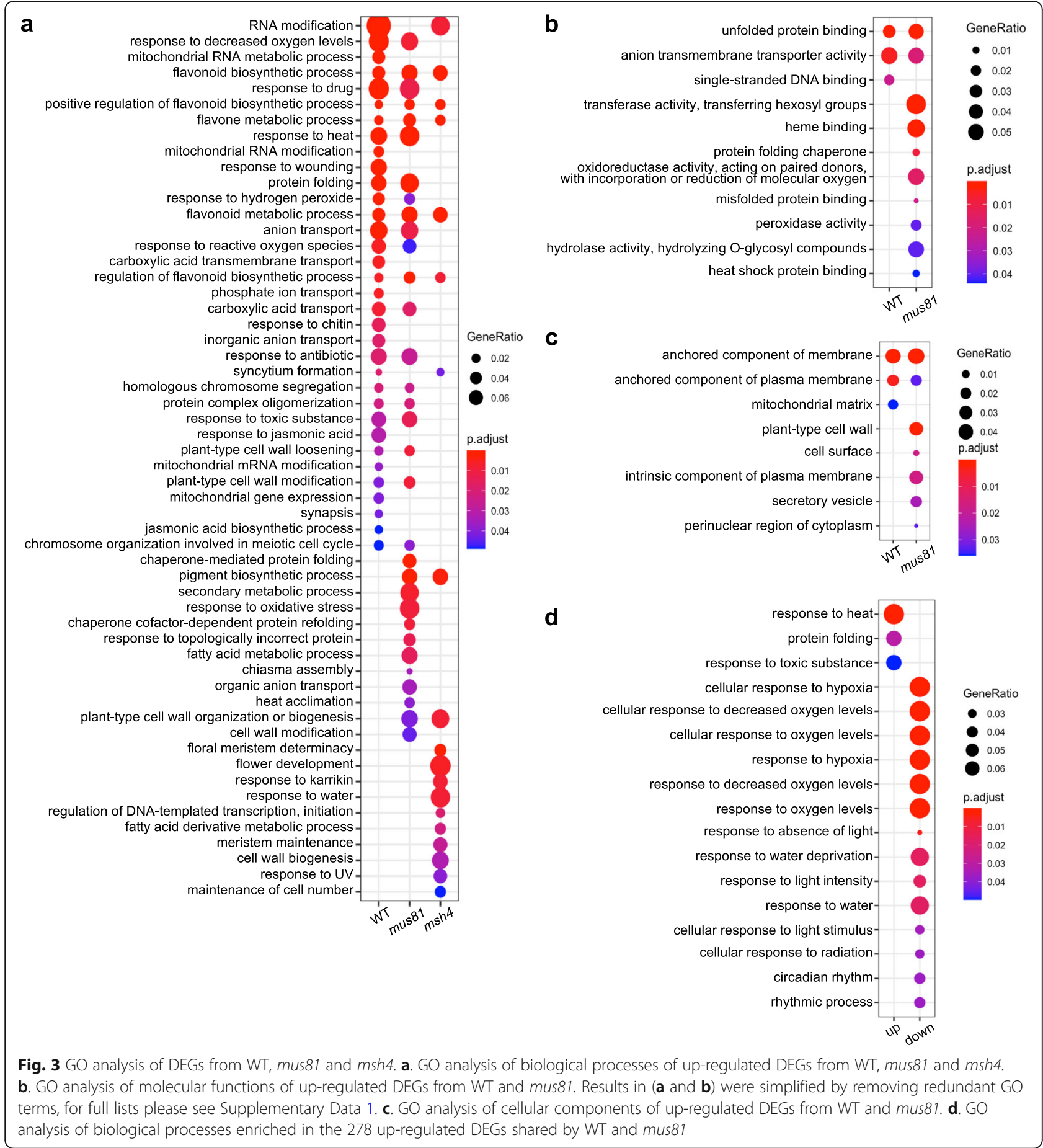

proper interhomolog interaction including chromosome pairing, synapsis and recombination [16], as well as ensuring crossover interference [17]. $A S Y 2$ is a putative functional homolog of $A S Y 1$ [15]. No meiosis-related DEGs were found among the 384 down-regulated DEGs shared by WT and mus81 (Supplementary Data 3). Interestingly, 4 genes are up-regulated in all three genotypes: the meiosis- specific cyclin-like gene $S D S$ [18], meiosis-specific recombinase DMC1 [19], meiosis-specific cohesion complex component SYN1/REC8 [20], and SWI1 which functions in meiotic sister chromatid cohesion [21] (Supplementary Data 3). However, none of the 14 known meiotic genes involved in either the Type I or Type II CO pathways were differentially expressed (Table 1). The apparent up- 


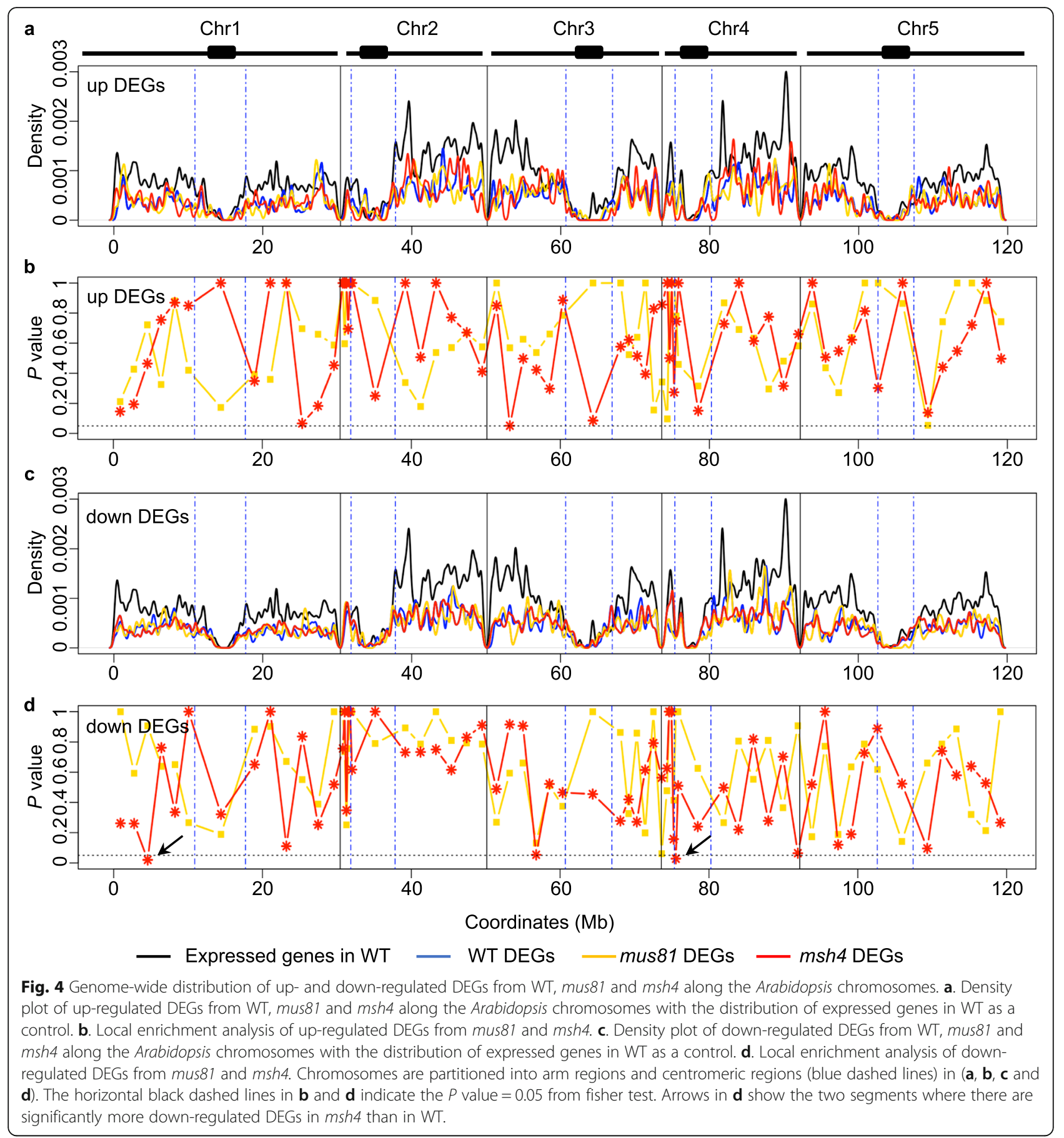

regulation of $\mathrm{MSH} 4$ in the msh4 mutant background at $28^{\circ} \mathrm{C}$ is most likely the result of the truncated $m s h 4$ being driven by the $35 \mathrm{~S}$ promoter carried by the T-DNA transgene insertion in this allele (Supplementary Figure 3B). Interestingly, we found one of the 21 Arabidopsis Heat Stress transcription Factor (HSF) genes, AtHSFA2 (AT2G26150) [22], is among the 278 DEGs up-regulated in WT and mus81 but not msh4 (Supplementary Data 4). In addition, AtHSFB2a (AT5G62020) and AtHSFA3 (AT5G03720) are up-regulated in WT, and AtHSFB1 (AT4G36990) is up-regulated in mus81. Surprisingly, no HSF transcription factors are up-regulated in msh4 (Supplementary Data 4). Further experimental work is needed to understand the potential interaction of the heat shock transcriptional regulatory networks and the meiotic recombination machinery. 


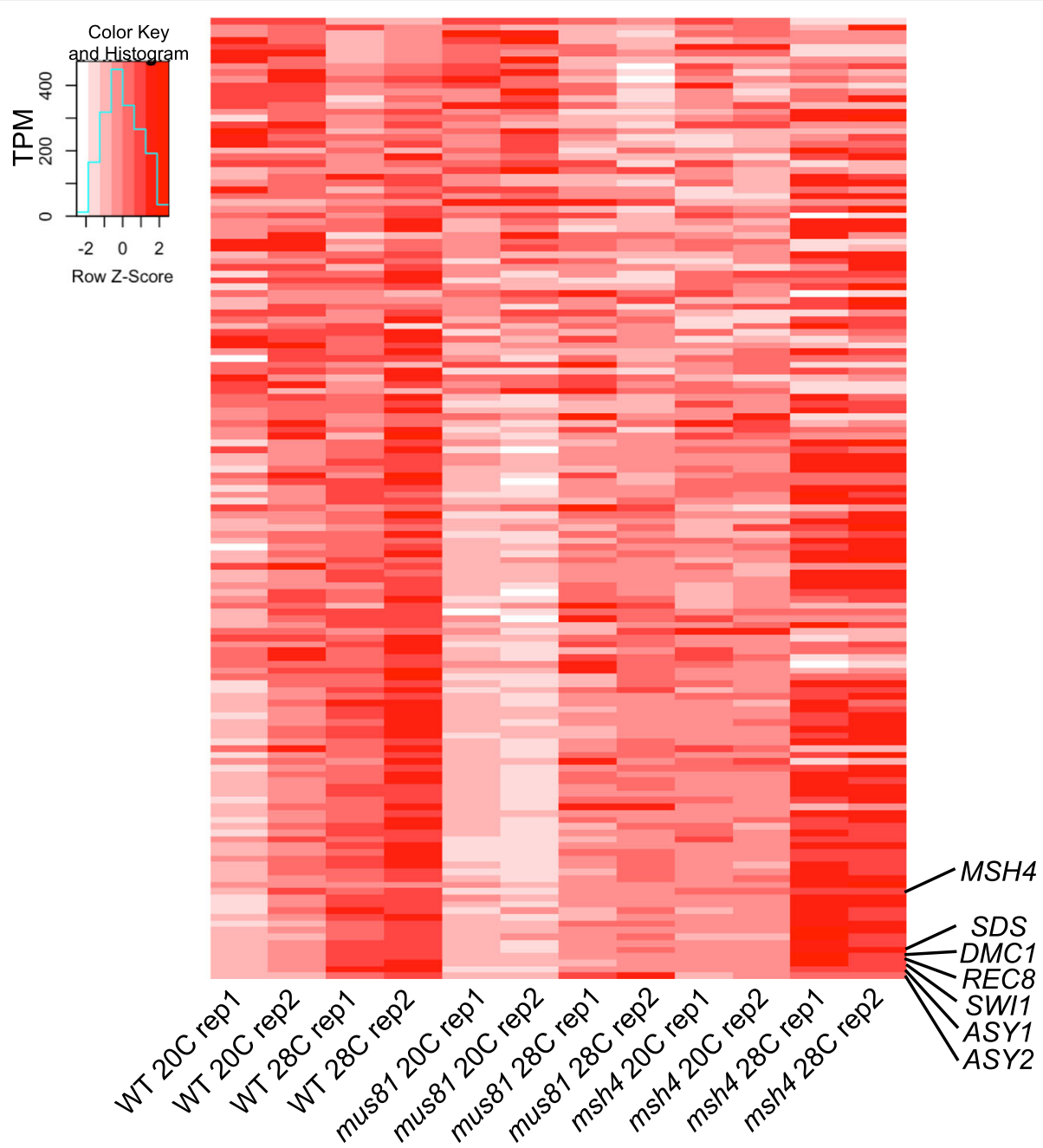

Fig. 5 Heatmap of gene expression data from 148 gene with previously defined meiotic functions from WT, mus 81 and msh4 plants grown at $20^{\circ} \mathrm{C}$ and $28^{\circ} \mathrm{C}$. The ranges of colors in the heatmap represent lower (white) to higher (dark red) relative expression of each gene (rows) in each sample (columns)

\section{Novel anther-specific transcripts associated with A-rich promoter motifs}

Our RNA-seq analysis of anthers identified 51 novel transcripts that had not been previously annotated in the Arabidopsis genome (Supplementary Data 5). 39\% of the novel transcripts have relatively low TPM values between 1 and 5, 14\% have TPMs of 5-10 (Fig. 6a) similar to previously annotated genes, $35 \%$ of which have TPMs of 1-10 (Fig. 1c). The novel transcripts are short with a median length of $809 \mathrm{bp}$ compared to $1787 \mathrm{bp}$ from the 38,194 annotated genes (Fig. 6b). However, 26 (46\%) of the novel transcripts have 2 exons, 14 (25\%) have 1 , and $8(14 \%)$ have 4 exons, compared to annotated genes in which the most abundant group (21\%) has only one exon (Fig. 6c). Comparison of the transcripts expressed at $20^{\circ} \mathrm{C}$ and $28{ }^{\circ} \mathrm{C}$ in the different genotypes reveals Differentially Expressed Novel Transcripts (DENTs) which include 5, 3 and 7 up-regulated and 6, 2 and 6 down- regulated DENTs from WT, mus81 and msh4, respectively (Fig. 6d). Noticeably, all DENTs in mus81 are shared by WT (Fig. 6d), which is consistent with the previous observations that the transcriptome profiles of WT and mus81 are more similar compared to msh4.

The loci encoding the novel transcripts are not preferentially partitioned into euchromatin or heterochromatin (Fisher's Exact Test, $P$ value $=0.18$ ) (Fig. 6e). However, we wondered whether they are expressed in a tissue-specific manner. To address this question, we generated two RNA-seq datasets from WT leaf tissue and compared them to the anther datasets (Supplementary Table 1). We found that $20(40 \%)$ of the 51 novel transcripts are anther-specific and 5 more are preferentially expressed in the anther when comparing $20^{\circ} \mathrm{C}$ anther datasets to WT leaves (Fig. 6e; Supplementary Data 5). Like the larger novel transcript set, the 20 anther- 
Table 1 Expression value (TPM) of genes in meiotic recombination type I and type II pathways

\begin{tabular}{|c|c|c|c|c|c|c|c|c|c|c|c|c|c|}
\hline Gene name & Alias & $\begin{array}{l}\text { WT } \\
20 C \\
\text { rep1 }\end{array}$ & $\begin{array}{l}\text { WT } \\
20 C \\
\text { rep2 }\end{array}$ & $\begin{array}{l}\text { WT } \\
28 C \\
\text { rep1 }\end{array}$ & $\begin{array}{l}\text { WT } \\
28 C \\
\text { rep2 }\end{array}$ & $\begin{array}{l}\text { mus81 } \\
20 \mathrm{C} \\
\text { rep1 }\end{array}$ & $\begin{array}{l}\text { mus81 } \\
20 \mathrm{C} \\
\text { rep2 }\end{array}$ & $\begin{array}{l}\text { mus81 } \\
28 \mathrm{C} \\
\text { rep1 }\end{array}$ & $\begin{array}{l}\text { mus81 } \\
28 \mathrm{C} \\
\text { rep2 }\end{array}$ & $\begin{array}{l}m s h 4 \\
20 C \\
\text { rep1 }\end{array}$ & $\begin{array}{l}\text { msh4 } \\
20 \mathrm{C} \\
\text { rep2 }\end{array}$ & $\begin{array}{l}m s h 4 \\
28 C \\
\text { rep1 }\end{array}$ & $\begin{array}{l}m s h 4 \\
28 C \\
\text { rep2 }\end{array}$ \\
\hline \multicolumn{14}{|c|}{ Type I CO pathway regulators } \\
\hline AT4G17380 & MSH4 & 7.3 & 9.8 & 9.0 & 10.4 & 5.3 & 4.6 & 5.8 & 7.4 & 8.6 & 9.6 & 38.0 & 50.1 \\
\hline AT3G20475 & MSH5 & 20.4 & 25.2 & 20.4 & 24.5 & 14.6 & 12.3 & 19.0 & 20.3 & 23.3 & 20.5 & 16.5 & 16.5 \\
\hline AT5G48390 & ZIP4 & 4.8 & 5.7 & 7.2 & 7.9 & 3.1 & 2.9 & 4.8 & 5.9 & 5.3 & 4.9 & 6.6 & 6.4 \\
\hline AT3G27730 & $\begin{array}{l}R C K \\
\text { MER3 }\end{array}$ & 4.8 & 8.1 & 6.3 & 8.2 & 3.0 & 2.7 & 4.6 & 5.1 & 7.6 & 5.5 & 8.0 & 7.8 \\
\hline AT1G12790 & PTD & 15.1 & 15.4 & 14.5 & 17.2 & 8.9 & 12.3 & 9.9 & 11.8 & 14.3 & 9.7 & 12.9 & 13.4 \\
\hline AT5G52290 & $\begin{array}{l}\text { SHOC1/ } \\
\text { ZIP2 }\end{array}$ & 1.3 & 2.0 & 2.5 & 3.4 & 1.0 & 1.0 & 1.2 & 1.6 & 1.8 & 1.6 & 3.1 & 3.1 \\
\hline AT1G53490 & HEl1O & 6.9 & 9.2 & 9.7 & 10.7 & 26.5 & 28.1 & 27.6 & 22.9 & 7.4 & 6.3 & 12.6 & 11.5 \\
\hline AT4G09140 & MLH1 & 26.5 & 26.8 & 26.3 & 29.6 & 21.2 & 21.0 & 23.0 & 21.5 & 24.8 & 26.4 & 27.2 & 26.2 \\
\hline AT4G35520 & MLH3 & 6.8 & 7.5 & 6.8 & 9.1 & 5.8 & 5.9 & 9.0 & 6.8 & 7.1 & 8.1 & 4.8 & 5.9 \\
\hline AT1G63770 & MPA1 & 122.1 & 115.0 & 116.4 & 128.4 & 105.0 & 96.5 & 111.5 & 114.4 & 117.6 & 120.3 & 124.8 & 119.7 \\
\hline Type II CO path & hway reg & ulators & & & & & & & & & & & \\
\hline AT4G30870 & MUS81 & 22.6 & 24.0 & 25.0 & 30.2 & 4.5 & 5.4 & 5.0 & 4.9 & 21.0 & 20.2 & 17.2 & 19.1 \\
\hline AT2G21800 & EMETA & 4.4 & 5.6 & 7.5 & 7.2 & 4.7 & 5.7 & 7.0 & 5.6 & 4.7 & 5.5 & 8.4 & 9.2 \\
\hline AT2G22140 & EME1B & 3.1 & 4.2 & 6.0 & 5.6 & 3.8 & 3.0 & 2.9 & 3.2 & 3.7 & 3.4 & 5.8 & 5.4 \\
\hline AT4G14970 & FANCD2 & 5.9 & 8.3 & 10.7 & 12.1 & 5.2 & 4.6 & 7.1 & 7.9 & 7.8 & 7.6 & 12.9 & 14.1 \\
\hline
\end{tabular}

specific transcripts are not preferentially partitioned into euchromatin or heterochromatin (Fisher's Exact Test, $P$ value $=0.29$ ) (Fig. 6e). To test whether these antherspecific novel transcripts are regulated by similar cisregulatory elements we used MEME to search for conserved DNA motifs [23]. 43 (84\%) of the novel transcripts had an upstream A-rich motif in their putative promoter regions, including 90\% (18 from 20) of the anther-specific novel transcripts $(E$ value $=8.8 \mathrm{e}-047)$ (Fig. 6f). These A-rich motifs have been reported to be associated with open chromatin structure and meiotic recombination hotspots [24-27], suggesting that the anther-specific novel transcripts may be expressed in response to a change in chromatin state associated with meiotic recombination. Moreover, of the 51 novel transcripts we identified 9 can potentially code for proteins (Supplementary Data 6). Of those MSTR G.16271, resembles a Danio rerio gene that encodes the guanine nucleotide exchange factor subunit RIC1 (Fig. 6g).

\section{Discussion}

Meiotic recombination is genetically regulated but also responds to external factors like temperature, drought and nutritient availability $[5,6]$. However, the underlining molecular mechanisms that facilitate the regulation of meiotic recombination are still largely uncharacterized. Previous studies from our lab and others have shown that moderate heat stress results in an increase in Type I COs in Arabidopsis [2, 3]. Here we analyzed the transcriptomes of Type I and Type II CO pathways mutants msh4 and mus81, under normal and heat stress growth conditions, and found that fewer genes are expressed after heat stress regardless of genotype (Fig. 1a). Other meiotic changes have also been observed in response to heat stress including a negative correlation between Arabidopsis synaptonemal complex (SC) length and temperature [3]. The Type I pathway mutant $m s h 4$ has the fewest expressed genes at $20^{\circ} \mathrm{C}$, and $3.3 \%$ fewer at $28^{\circ} \mathrm{C}$ (Fig. 1a). Consistent with this observation, we identified more down-regulated DEGs in msh4 as 


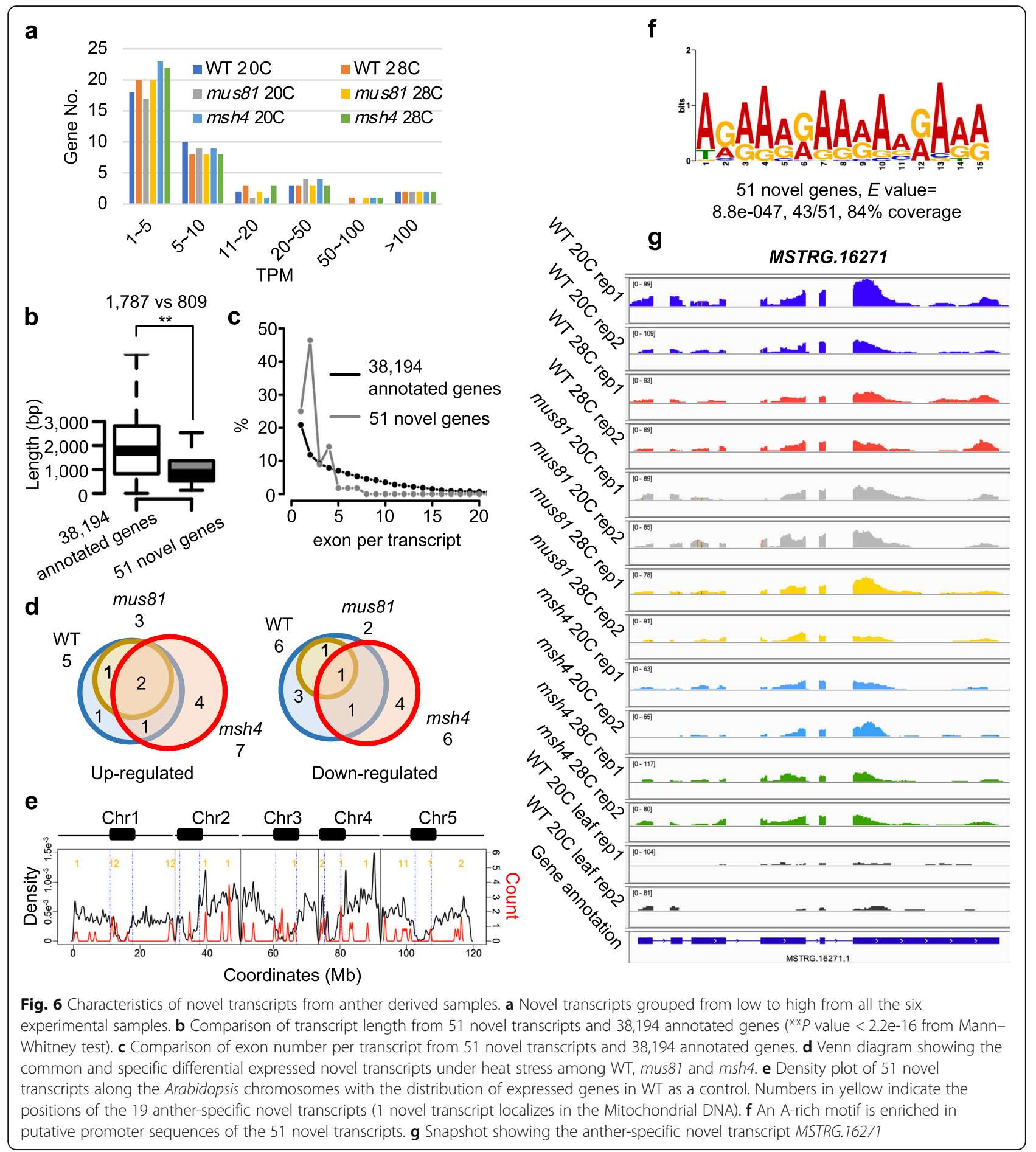

compared to WT or mus81 (Fig. 2b). It will be interesting to know whether the transcriptional changes we have documented are directly connected, on a mechanistic level, with regulating $\mathrm{SC}$ length or $\mathrm{CO}$ number.

We observed that $A S Y 1$ and $A S Y 2$ are the only previously characterized meiotic recombination-associated genes that are significantly up-regulated in WT and mus81 but not in msh4 (Fig. 2). This is consistent with previous observations that barley $A S Y 1$ is prematurely up-regulated in early stage anthers under hightemperature conditions [28]. MUS81 mediates the CO interference insensitive (Type II) meiotic pathway, while msh4 is required for the interference sensitive (Type I) pathway. This suggests that $A S Y 1$ and $A S Y 2$ may 
potentially function in tuning the balance between meiotic recombination pathways in response to external cues like heat stress. In Arabidopsis, ASY1 is known to antagonize telomere-led recombination in a dosagedependent manner [17]. In autotetraploid Arabidopsis arenosa, different ASY1 alleles influence meiotic chromosome interactions, morphology and axis length [29], and $A S Y 1$ is among the key axis component genes that are under strong selection [30]. These results are consistent with the hypothesis that the regulation of ASY1 gene expression influences meiotic recombination levels. However, recent studies in Arabidopsis reported no change in ASY1 immunostaining in wild type meiocytes after $30-32{ }^{\circ} \mathrm{C}$ heat shock for $24 \mathrm{~h}$ [4], but partially compromised localization under $36-38^{\circ} \mathrm{C}$ extreme heat stress conditions [31]. It is important to note that the regulatory responses to moderate environmental stresses may not be reflected in more extreme conditions which can instead result in meiotic failure.

We identified 51 novel transcripts from our anther derived RNA-seq datasets (Fig. 6) including 20 that are anther-specific (Fig. 6) and 9 that have open reading frames encoding more than 100 amino acids (Supplementary Data 6). Arabidopsis thaliana has a mature and well annotated genome but the latest Arabidopsis annotation (Araport11) was constructed from 11 tissues that did not specifically include early stage floral buds that contain anthers undergoing meiosis (though later stage anthers were included) [32]. Similarly, the libraries used for the annotation did not include plants that had been subjected to abiotic stress conditions. Thus, it is not unreasonable that we identified several previously unannotated transcripts since we were examing an underrepresented tissue type in specific environmental conditions. Interestingly, we found that the upstream putative promoter regions of the loci encoding the 51 novel transcripts are enriched for a known meiotic recombination hotspot-associated A-rich motif (Fig. 6f). The relevance of this finding may be somewhat tempered by the fact that A-rich elements are abundant in the genome and are also significantly enriched in the set of all known gene promoters $(E$ value $=5.3 \mathrm{e}-103)$ (Supplementary Figure 4). At the very least, the association of the A-rich motif with these 51 novel transcript loci suggests that they are bona fide genes. More speculatively, since the A-rich motif has been reported to be associated with open chromatin structure and meiotic recombination hotspots [24-27], it is possible that these novel transcripts are up-regulated as the result of changes in chromatin structure associated with meiotic recombination.

\section{Conclusions}

By comparing thetranscriptional profiles of Arabidopsis wild type and meiotic recombination mutants under normal and heats-stress conditions, we show that mutants in the Type II meiotic recombination pathway (mus81) are more similar to WT than mutants in the Type I pathway (msh4). The chromosomal axis protein ASY1 is up-regulated in both WT and mus81, but not msh4. We also describe 51 novel transcripts that are expressed in meiotic tissues, including several that are differentially expressed under heat stress conditions. Interestingly, the loci encoding these novel transcripts are enriched for a previously defined A-rich DNA motif that have been associated with open chromatin and meiotic recombination hotspots. We believe this work will be of interest to the meiosis community.

\section{Methods}

\section{Plant materials and growth conditions}

Arabidopsis thaliana lines including wild type Col-0, mus81 (SALK_107515) and msh4 (SALK_136296) used in this study were obtained from the Arabidopsis Biological Resource Center. Plants were grown in soil under long day ( $16 \mathrm{~h}$ light and $8 \mathrm{~h}$ dark) growth room conditions at $20^{\circ} \mathrm{C}$. For the heat treatment, flowering plants were placed in a $28{ }^{\circ} \mathrm{C}$ growth chamber for 5 days. Anthers at stage 4-7 [14] were collected using a dissecting microscope on the fifth day, frozen in liquid nitrogen and stored in $-80{ }^{\circ} \mathrm{C}$ for later use. Genotyping primers are provided in Supplementary Table 3.

\section{Transcriptome sequencing, data collection, and analysis}

Total RNA was extracted using Trizol (Thermo Fisher Scientific) following the manufacturer's protocol from stage 4-7 anthers collected as described above. mRNASeq libraries were constructed using TruSeq RNA Library Preparation Kits (Illumina). Paired-end sequencing was performed with an Illumina Hiseq 3000. Retrieved raw reads were trimmed using BBMap (version 38.82, Bushnell B., https://sourceforge.net/projects/bbmap/) to remove adapters. Whole genome sequence and Araport11 annotation were downloaded from TAIR10 (https://www.Arabidopsis.org/). Filtered reads were mapped using TopHat2 [33] then analyzed using Stringtie [34] and DESeq2 [35]. TPM values of each gene from all conditions were calculated using Stringtie with the parameter "-A" [34]. An expressed gene was defined using a cut off of $\geq 1$ TPM in both biological replicates. Differentially expressed genes were analysed using DESeq2 using the following criteria: $\log 2 \mathrm{fdc}>1$ or $<-1$, $\mathrm{q}$ value $<0.05$. GO analyses were performed in $\mathrm{R}$ using the clusterProfiler package [36]. GO term enrichment plot results were simplified with the command "simplify" $(x$, cutoff $=0.7$, by $=$ "p.adjust", select_fun $=$ min $)$. Illustrations of gene expression profiles were plotted using the Integrative Genomics Viewer (IGV 2.8.2). Pearson's product-moment correlation tests were performed in $\mathrm{R}$. 
Chromosome local DEGs enrichment tests were calculated using fisher test in R.

\section{DNA motif discovery}

We defined the $1000 \mathrm{bp}$ sequence upstream of each novel transcript as its putative promoter. Potential cisregulatory element or conserved DNA motif discovery of the 51 putative promoter sequences was analyzed using MEME (5.1.1) [23] with the default parameters with the following exceptions: -mod anr - minw 5 -maxw 30 -revcomp.

\section{Novel transcript analysis}

Novel transcripts were discovered using Stringtie with a parameter "-m 30" [34]. We used a cut off of $\geq 1$ TPM in both biological replicates in the WT 20C, WT 28C, mus81 20C, mus $8128 \mathrm{C}$, msh $420 \mathrm{C}$ or msh4 $28 \mathrm{C}$ datasets to define the novel transcripts. Anther-specific novel transcripts were defined as those that had $\geq 1$ TPM in both anther datasets, but $<1$ TPM in both biological replicates from the leaves. Novel preferentially expressed anther transcripts were defined as those that had $>1$ TPM in both biological replicates in leaves and significantly higher TPM values in WT 20C, mus $8120 \mathrm{C}$ and, msh4 20C compared to leaves using DESeq2 [35], $\log 2 \mathrm{fdc}>1$, q value $<0.05$. Candidate coding regions within transcript sequences and potential protein sequences were identified by using TransDecoder with its default setting (https://github.com/TransDecoder/ TransDecoder/). Only protein sequences larger than 100 amino acid were retained.

\section{Abbreviations}

BP: Biological process; CC: Cellular component; CO: Crossover;

DEG: Differential expressed gene; DENT: Differentially Expressed Novel Transcript; HSF: Heat stress transcription factor; MF: Molecular function; MMR: Mismatch repair; SC: Synaptonemal complex; TPM: Transcripts Per kilobase Million; WT: Wildtype

\section{Supplementary Information}

The online version contains supplementary material available at https://doi. org/10.1186/s12864-021-07497-2.

Additional file 1 : Supplementary Table 1. Characteristics of the 14

RNA-Seq datasets used in the study.

Additional file $\mathbf{2}$ : Supplementary Table 2. Pearson's product moment correlation coefficients ( $r$ ) between pairs of samples used in this study.

Additional file 3 : Supplementary Table 3. Genotyping primers used in this study.

Additional file 4 : Supplementary Figure 1. Intersection of expressed genes among all 6 samples in $\mathbf{a}$, and expressed gene numbers grouped from low to high of the 63 genes only expressed in WT and mus81 at $28^{\circ} \mathrm{C}$ in $\mathbf{b}$

Additional file 5 : Supplementary Figure 2. GO analysis of biological processes $\mathbf{a}$, molecular functions $\mathbf{b}$ and cellular components $\mathbf{c}$ of downregulated DEGs from WT, mus 81 and msh4.
Additional file $\mathbf{6}$ : Supplementary Figure 3. Gene expression of ASY2 and MSH4 under heat stress in WT, mus81 and msh4. a Snapshot showing that ASY2 is an up-regulated DEG in WT and mus81 but not in msh4. b Snapshot showing atypical up-regulated MSH4 in msh4 but not in WT and mus81. c Expression of ASY2 from WT, mus81 and msh4 grown under $20^{\circ} \mathrm{C}$ and $28^{\circ} \mathrm{C}$ conditions.

Additional file 7 : Supplementary Figure 4. A-rich motif enriched in the promoter sequences of 51 randomly selected genes from Arabidopsis Araport11 annotation set.

Additional file 8 : Supplementary Data 1. Enriched GO terms from up- and down-regulated DEGs in WT, mus 81 and msh4 after heat stress.

Additional file 9 : Supplementary Data 2. Enriched GO terms from 278 up- and 384 down-regulated DEGs found in WT and mus81 after heat stress but not in msh4.

Additional file 10 : Supplementary Data 3. Expression data from 148 genes with previously described meiotic functions in WT, mus81 and msh4 at $20^{\circ} \mathrm{C}$ and $28^{\circ} \mathrm{C}$.

Additional file 11 : Supplementary Data 4. Expression data from the 21 Arabidopsis HSF genes in WT, mus 81 and msh4 at $20^{\circ} \mathrm{C}$ and $28^{\circ} \mathrm{C}$.

Additional file $\mathbf{1 2}$ : Supplementary Data 5. 51 novel transcripts described in this study.

Additional file 13 : Supplementary Data 6. Potential Open Reading Frames (ORFs) of the 9 novel genes.

\section{Acknowledgements}

We would like to thank the Genergy Bio-Technology (Shanghai) Co., Ltd. for the help in library preparation and sequencing.

\section{Authors' contributions}

$H W, W Y$, and GPC designed the research. HW collected the data. JH analyzed the data. JH, WY, and GPC wrote the manuscript. All authors contributed to the article and approved the submitted version.

\section{Funding}

This work was supported by the grant from the US National Science Foundation (IOS-1844264) to GPC and the National Science Foundation of China (31925005) to WY.

Availability of data and materials

The datasets supporting the conclusions of this article are available in the NCBI Sequence Read Archive (SRA) repository under the project name PRJNA679774 (https://www.ncbi.nlm.nih.gov/bioproject/ PRJNA679774). All other datasets generated for this study are included in the article/ Supplementary Material, further inquiries can be directed to the corresponding authors.

\section{Declarations}

Ethics approval and consent to participate Not applicable.

\section{Consent for publication}

Not applicable.

\section{Competing interests}

The authors declare that they have no competing interests.

\section{Author details}

${ }^{1}$ Department of Biology and the Integrative Program for Biological and Genome Sciences, University of North Carolina at Chapel Hill, Chapel Hill, North Carolina, USA. ${ }^{2}$ State Key Laboratory of Genetic Engineering and Collaborative Innovation Center of Genetics and Development, Ministry of Education Key Laboratory of Biodiversity Sciences and Ecological Engineering, Institute of Plant Biology, School of Life Sciences, Fudan University, Shanghai, China. '²ineberger Comprehensive Cancer Center, University of North Carolina School of Medicine, Chapel Hill, North Carolina, USA. 
Received: 2 December 2020 Accepted: 25 February 2021

Published online: 12 March 2021

\section{References}

1. Wang Y, Copenhaver GP. Meiotic recombination: mixing it up in plants. Annu Rev Plant Biol. 2018;69:577-609.

2. Modliszewski JL, Wang H, Albright AR, Lewis SM, Bennett AR, Huang J, et al. Elevated temperature increases meiotic crossover frequency via the interfering (Type I) pathway in Arabidopsis thaliana. PLoS Genet. 2018;14: e1007384

3. Lloyd A, Morgan C, Franklin C, Bomblies K. Plasticity of meiotic recombination rates in response to temperature in Arabidopsis. Genetics. 2018;208:1409-20.

4. De Storme N, Geelen D. High temperatures alter cross-over distribution and induce male meiotic restitution in Arabidopsis thaliana. Commun Biol. 2020; 3:187.

5. Modliszewski JL, Copenhaver GP. Meiotic recombination gets stressed out: CO frequency is plastic under pressure. Curr Opin Plant Biol. 2017;36:95-102.

6. Bomblies K, Higgins JD, Yant L. Meiosis evolves: adaptation to external and internal environments. New Phytol. 2015:208:306-23.

7. Phillips D, Jenkins G, Macaulay M, Nibau C, Wnetrzak J, Fallding D, et al. The effect of temperature on the male and female recombination landscape of barley. New Phytol. 2015;208:421-9.

8. Lei $X$, Ning Y, Eid Elesawi I, Yang K, Chen C, Wang C, et al. Heat stress interferes with chromosome segregation and cytokinesis during male meiosis in Arabidopsis thaliana. Plant Signal Behav. 2020;15:1746985.

9. Draeger T, Moore G. Short periods of high temperature during meiosis prevent normal meiotic progression and reduce grain number in hexaploid wheat (Triticum aestivum L.). Theor Appl Genet. 2017;130:1785-800.

10. Osman K, Higgins JD, Sanchez-Moran E, Armstrong SJ, Franklin FC. Pathways to meiotic recombination in Arabidopsis thaliana. New Phytol. 2011;190:523-44.

11. Higgins JD, Armstrong SJ, Franklin FC, Jones GH. The Arabidopsis MutS homolog AtMSH4 functions at an early step in recombination: evidence for two classes of recombination in Arabidopsis. Genes Dev. 2004;18:2557-70.

12. Berchowitz LE, Francis KE, Bey AL, Copenhaver GP. The role of AtMUS81 in interference-insensitive crossovers in A. thaliana. PLoS Genet. 2007;3:e132.

13. Jackson N, Sanchez-Moran E, Buckling E, Armstrong SJ, Jones GH, Franklin FC. Reduced meiotic crossovers and delayed prophase I progression in AtMLH3-deficient Arabidopsis. EMBO J. 2006;25:1315-23.

14. Sanders PM, Bui AQ, Weterings K, Mclntire KN, Hsu YC, Lee PY, et al. Anther developmental defects in Arabidopsis thaliana male-sterile mutants. Sex Plant Reprod. 1999;11:297-322.

15. Caryl AP, Armstrong SJ, Jones GH, Franklin FCH. A homologue of the yeast HOP1 gene is inactivated in the Arabidopsis meiotic mutant asy1. Chromosoma. 2000;109:62-71.

16. Zickler D, Kleckner N. Meiotic chromosomes: integrating structure and function. Annu Rev Genet. 1999;33:603-754.

17. Lambing C, Kuo PC, Tock AJ, Topp SD, Henderson IR. ASY1 acts as a dosage-dependent antagonist of telomere-led recombination and mediates crossover interference in Arabidopsis. Proc Natl Acad Sci U S A. 2020;117: 13647-58.

18. Azumi Y, Liu D, Zhao D, Li W, Wang G, Hu Y, et al. Homolog interaction during meiotic prophase I in Arabidopsis requires the SOLO DANCERS gene encoding a novel cyclin-like protein. EMBO J. 2002;21:3081-95.

19. Couteau F, Belzile F, Horlow C, Grandjean O, Vezon D, Doutriaux MP. Random chromosome segregation without meiotic arrest in both male and female meiocytes of a dmc1 mutant of Arabidopsis. Plant Cell. 1999;11:1623-34.

20. Bai X, Peirson BN, Dong F, Xue C, Makaroff CA. Isolation and characterization of SYN1, a RAD21-like gene essential for meiosis in Arabidopsis. Plant Cell. 1999;11:417-30.

21. Yang C, Hamamura Y, Sofroni K, Bower F, Stolze SC, Nakagami H, et al. SWIT $\mathrm{CH}$ 1/DYAD is a WINGS APART-LIKE antagonist that maintains sister chromatid cohesion in meiosis. Nat Commun. 2019;10:1755.

22. Guo M, Liu JH, Ma X, Luo DX, Gong ZH, Lu MH. The plant heat stress transcription factors (HSFs): structure, regulation, and function in response to abiotic stresses. Front Plant Sci. 2016;7:114.

23. Bailey $\mathrm{TL}$, Elkan C. Fitting a mixture model by expectation maximization to discover motifs in biopolymers. Proc Int Conf Intell Syst Mol Biol. 1994:2:28-36.

24. Choi K, Zhao X, Kelly KA, Venn O, Higgins JD, Yelina NE, et al. Arabidopsis meiotic crossover hot spots overlap with H2A.Z nucleosomes at gene promoters. Nat Genet. 2013;45:1327-36.
25. Wijnker E, Velikkakam James G, Ding J, Becker F, Klasen JR, Rawat V, et al. The genomic landscape of meiotic crossovers and gene conversions in Arabidopsis thaliana. Elife. 2013;2:e01426.

26. Shilo S, Melamed-Bessudo C, Dorone Y, Barkai N, Levy AA. DNA crossover motifs associated with epigenetic modifications delineate open chromatin regions in Arabidopsis. Plant Cell. 2015;27:2427-36.

27. Huang J, Wang C, Wang H, Lu P, Zheng B, Ma H, et al. Meiocyte-specific and AtSPO11-1-dependent small RNAs and their association with meiotic gene expression and recombination. Plant Cell. 2019;31:444-64.

28. Oshino T, Abiko M, Saito R, Ichiishi E, Endo M, Kawagishi-Kobayashi M, et al. Premature progression of anther early developmental programs accompanied by comprehensive alterations in transcription during hightemperature injury in barley plants. Mol Gen Genomics. 2007;278:31-42.

29. Morgan C, Zhang H, Henry CE, Franklin FCH, Bomblies K. Derived alleles of two axis proteins affect meiotic traits in autotetraploid Arabidopsis arenosa. Proc Natl Acad Sci U S A. 2020;117:8980-8.

30. Yant L, Hollister JD, Wright KM, Arnold BJ, Higgins JD, Franklin FCH, et al. Meiotic adaptation to genome duplication in Arabidopsis arenosa. Curr Biol. 2013:23:2151-6.

31. Ning Y, Liu Q, Wang C, Qin E, Wu Z, Wang M, et al. Heat stress interferes with formation of double-strand breaks and homology synapsis. Plant Physiol. 2021;0:kiab012

32. Cheng CY, Krishnakumar V, Chan AP, Thibaud-Nissen F, Schobel S, Town CD. Araport11: a complete reannotation of the Arabidopsis thaliana reference genome. Plant J. 2017;89:789-804.

33. Trapnell C, Roberts A, Goff L, Pertea G, Kim D, Kelley DR, et al. Differential gene and transcript expression analysis of RNA-seq experiments with TopHat and Cufflinks. Nat Protoc. 2012;7:562-78.

34. Pertea M, Pertea GM, Antonescu CM, Chang TC, Mendell JT, Salzberg SL. StringTie enables improved reconstruction of a transcriptome from RNA-seq reads. Nat Biotechnol. 2015:33:290-5.

35. Love Ml, Huber W, Anders S. Moderated estimation of fold change and dispersion for RNA-seq data with DESeq2. Genome Biol. 2014;15:550.

36. Yu G, Wang LG, Han Y, He QY. clusterProfiler: an R package for comparing biological themes among gene clusters. Omics. 2012;16:284-7.

\section{Publisher's Note}

Springer Nature remains neutral with regard to jurisdictional claims in published maps and institutional affiliations.

Ready to submit your research? Choose BMC and benefit from

- fast, convenient online submission

- thorough peer review by experienced researchers in your field

- rapid publication on acceptance

- support for research data, including large and complex data types

- gold Open Access which fosters wider collaboration and increased citations

- maximum visibility for your research: over $100 \mathrm{M}$ website views per year

At $\mathrm{BMC}$, research is always in progress.

Learn more biomedcentral.com/submissions 